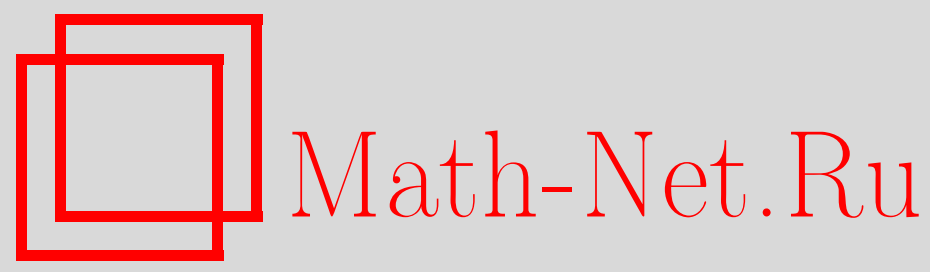

О. А. Горкуша, Минимальные базисы трехмерных полных решеток, Матем. заметки, 2001, том 69, выпуск 3, 353-362

DOI: https://doi.org/10.4213/mzm509

Использование Общероссийского математического портала Math-Net.Ru подразумевает, что вы прочитали и согласны с пользовательским соглашением http://www. mathnet.ru/rus/agreement

Параметры загрузки:

IP: 34.239 .49 .27

26 апреля 2023 г., 18:16:36

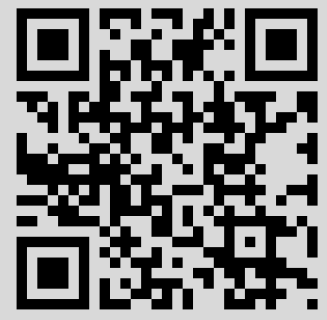




\section{МИНИМАЛЬНЫЕ БАЗИСЫ ТРЕХМЕРНЫХ ПОЛНЫХ РЕШЕТОК}

\section{О. А. Горкуша}

В работе строятся так называемые "минимальные базисы" в смысле Минковского для трехмерных полных решеток.

Библиография: 4 названия.

Обозначения. 1 . Натуральное $s=2,3, \ldots-$ размерность $\mathbb{R}^{s}$.

2. Для $x=\left(x_{1}, \ldots, x_{s}\right) \in \mathbb{R}^{s}$

$$
\mathscr{P}(x)=\left\{x^{\prime} \in \mathbb{R}^{s}|| x_{i}^{\prime}|\leqslant| x_{i} \mid, i=1, \ldots, s\right\} .
$$

3. Для непустого конечного подмножества $T$ в $\mathbb{R}^{s}$

$$
|T|_{i}=\max \left\{\left|x_{i}\right| \mid x=\left(x_{1}, \ldots, x_{i}, \ldots, x_{s}\right) \in T\right\}, \quad \mathscr{P}(T)=\mathscr{P}\left(|T|_{1}, \ldots,|T|_{s}\right) .
$$

4. $\mathfrak{L}_{s}(\mathbb{R})$ - множество всех полных решеток в $\mathbb{R}^{s}$ (в дальнейшем просто решеток).

5. $\mathfrak{M}(\Gamma)$ - множество всех относительных минимумов решетки, состоящих из узлов $\gamma \in \Gamma^{\prime}=\Gamma \backslash\{0, \ldots, 0\}$, для которых не существует других ненулевых узлов $\gamma^{\prime}$ из $\Gamma$ со строгим включением $\mathscr{P}\left(\gamma^{\prime}\right) \subset \mathscr{P}(\gamma)$.

Введение. Напомним, что произвольная решетка $Г$ из $\mathfrak{L}_{s}(\mathbb{R})$ имеет вид

$$
\Gamma=\left\{m_{1} \gamma^{(1)}+\cdots+m_{s} \gamma^{(s)} \mid m_{1}, \ldots, m_{s} \in \mathbb{Z}\right\}
$$

с линейно независимыми узлами $\gamma^{(1)}, \ldots, \gamma^{(s)}$, составляющими целочисленньй базис $\Gamma$ (см. [1]). В работе [2] Вороной построил теорию “относительных минимумов" (термин принадлежит ему же) для двумерных и трехмерных решеток из класса

$$
\mathfrak{L}_{s}^{*}(\mathbb{R})=\left\{\Gamma \in \mathfrak{L}_{s}(\mathbb{R}) \mid \forall\left(\gamma_{1}, \ldots, \gamma_{s}\right) \in \Gamma^{\prime}, \gamma_{1} \neq 0, \ldots, \gamma_{s} \neq 0\right\} .
$$

Практически одновременно и независимо в заметке [3] Минковский рассмотрел тройки $S=\left\{\gamma^{(1)}, \gamma^{(2)}, \gamma^{(3)}\right\}$ линейно независимых узлов решетки $\Gamma$ из $\mathfrak{L}_{3}^{*}(\mathbb{R})$, которые удовлетворяют следующему условию минимальности: не существует ненулевых узлов $\gamma \in \Gamma \mathrm{c}$

$$
\left|\gamma_{1}\right|<|S|_{1}, \quad\left|\gamma_{2}\right|<|S|_{2}, \quad\left|\gamma_{3}\right|<|S|_{3}
$$

Он доказал, что любая такая тройка составляет базис решетки.

Вороной и Минковский ограничились изучением решеток из $\mathfrak{L}_{3}^{*}(\mathbb{R})$, поскольку их в первую очередь интересовали методы построения единиц в кубических числовых полях, 
обобщающие хорошо известный до них алгоритм непрерывных дробей для вычисления единиц квадратичных полей. Для решения задач целочисленного линейного программирования представляет интерес перенос этих конструкций на случай целочисленных решеток, не принадлежащих классу $\mathfrak{L}_{3}^{*}(\mathbb{R})$.

Для произвольной решетки $\Gamma \in \mathfrak{L}_{3}(\mathbb{R})$ в определении "минимальной системы" $S$ помимо условия (0.1) потребуем, чтобы $\gamma^{(1)}, \gamma^{(2)}, \gamma^{(3)}$ были относительными минимумами. Для решетки из $\mathfrak{L}_{3}^{*}(\mathbb{R})$ это требование следует из $(0.1)$.

Любая минимальная система из двух узлов произвольной решетки $\Gamma \in \mathfrak{L}_{2}(\mathbb{R})$ составляет базис $\Gamma$. Доказательство этого факта для случая $\Gamma \in \mathfrak{L}_{2}^{*}(\mathbb{R})$, предложенное Вороньм в [2], практически без изменений переносится на любые решетки из $\mathfrak{L}_{2}(\mathbb{R})$.

В настоящей работе для трехмерных решеток мы доказьваем следуюший результат.

Теорема 1. Пусть решетка Г порождается базисными узлами

$$
\gamma^{(1)}=\left(a_{1}, a_{2}, a_{3}\right), \quad \gamma^{(2)}=\left(a_{1}, 0, \frac{1}{2} c_{3}\right), \quad \gamma^{(3)}=\left(0,0, c_{3}\right)
$$

с положительными $a_{1}, a_{2}, c_{3}$ и $a_{3} \geqslant 0$. Тогда множество

$$
S=\left\{\gamma^{(1)}, 2 \gamma^{(2)}-\gamma^{(1)}-\gamma^{(3)}, \gamma^{(3)}\right\}=\left\{\left(a_{1}, a_{2}, a_{3}\right),\left(a_{1},-a_{2},-a_{3}\right),\left(0,0, c_{3}\right)\right\}
$$

- минимальная система тогда и только тогда, когда $4 a_{3}<c_{3}$.

Очевидно, что наборы узлов вида (0.3) порождают в Г подрешетку индекса 2. Все минимальные системы, которые получаются из (0.3) путем изменения порядка следования координат и знаков у некоторых из них (координат), назовем “исключительными". Разумеется, при таких трансформациях $S$ соответствующим образом меняется и сама решетка $\Gamma$.

Мы распространяем результат Минковского на произвольные решетки из $\mathfrak{L}_{3}(\mathbb{R})$ в следующем виде.

Теорема 2. Всякая минимальная система $S$ произвольной решетки $\Gamma \in \mathfrak{L}_{3}(\mathbb{R})$, отличная от “исключительной", составляет базис решетки Г.

\section{1. Минимальные "исключительные" системы.}

ДокАЗАТЕЛЬСТво ТЕОРЕмЫ 1 . Пусть $Г$ - решетка, порожденная базисньми узлами $(0.2)$ с $4 a_{3}<c_{3}$, и $S$ - система вида $(0.3)$. Любой узел решетки имеет вид

$$
\gamma=n \gamma^{(1)}+m \gamma^{(2)}+k \gamma^{(3)}=\left(a_{1}(n+m), n a_{2}, n a_{3}+c_{3}\left(k+\frac{1}{2} m\right)\right) .
$$

Если $\gamma$ лежит внутри $\mathscr{P}\left(|S|_{1},|S|_{2},|S|_{3}\right)$, то имеет место система неравенств

$$
\left\{\begin{array}{l}
a_{1}|n+m|<a_{1}, \\
a_{2}|n|<a_{2}, \\
\left|n a_{3}+\frac{1}{2} m c_{3}+k c_{3}\right|<c_{3} .
\end{array}\right.
$$

Значения $n=m=k=0$ являются единственным решением данной системы и при этом $\gamma=(0,0,0)$. Следовательно, вьполнено условие $(0.1)$ из определения минимальной системы. 
Очевидно, что $\gamma^{(3)}$ минимум. Рассмотрим систему неравенств

$$
\left\{\begin{array}{l}
a_{1}|n+m| \leqslant a_{1}, \\
a_{2}|n| \leqslant a_{2}, \\
\left|n a_{3}+\frac{1}{2} m c_{3}+k c_{3}\right| \leqslant a_{3}
\end{array}\right.
$$

относительно целых $n, m, k$. Поскольку $(n, m, k)$ и $(-n,-m,-k)$ одновременно являются решениями этой системы, достаточно рассмотреть случай с $n \geqslant 0$. Первьм двум неравенствам удовлетворяют только пары $(n, m)$ из

$$
\{(0,-1),(0,0),(1,-2),(1,-1),(1,0)\} .
$$

Так как $4 a_{3}<c_{3}$, то при $(n, m) \in\{(0,-1),(0,0)\}$ третье неравенство не имеет ненулевых решений. Для $n=1$ выполняется двухстороннее неравенство $-1 / 2-m / 2<k \leqslant$ $-m / 2$. Поэтому единственными ненулевыми решениями нашей системы являются тройки

$$
(n, m, k) \in\{(1,-2,1),(1,0,0)\} .
$$

Соответствуюшие им узлы решетки из (1.1) имеют вид $\left(-a_{1}, a_{2}, a_{3}\right),\left(a_{1}, a_{2}, a_{3}\right)$. Отсюда следует, что $\gamma^{(1)}$ и $2 \gamma^{(2)}-\gamma^{(1)}-\gamma^{(3)}$ являются минимумами. Итак, мы показали, что $S$ - минимальная система.

Докажем обратное утверждение теоремы 1 . Пусть $S$ - минимальная система вида (0.3) решетки $Г$ с базисом (0.2). Предположим, что $4 a_{3} \geqslant c_{3}$. Тогда узел

$$
\gamma=\gamma^{(1)}-\gamma^{(2)}=\left(0, a_{2}, a_{3}-\frac{1}{2} c_{3}\right)
$$

лежит внутри $\mathscr{P}\left(\gamma^{(1)}\right)$, что нарушает минимальность $\gamma^{(1)}$. Таким образом, предположение неверно. Теорема 1 полностью доказана.

Лемма 1. Пусть минимальная система $S$ некоторой решетки $Г$ состоит из узлов

$$
\gamma^{(1)}=\left(a_{1}, a_{2}, a_{3}\right), \quad \gamma^{(2)}=\left(a_{1},-b_{2},-b_{3}\right), \quad \gamma^{(3)}=\left(0,-c_{2}, c_{3}\right)
$$

с положстельным $a_{1}$ и неотрицательными $a_{2}, a_{3}, b_{2}, b_{3}, c_{2}, c_{3}$. Если для некоторой комбиначии знаков

$$
\frac{1}{2}\left( \pm \gamma^{(1)} \pm \gamma^{(2)} \pm \gamma^{(3)}\right) \in \Gamma
$$

то S - “исключительная" система.

ДокАЗАТЕЛЬСТво. Заметим, что если одна из комбинаций вида (1.3) является узлом решетки, то и все остальные также принадлежат этой решетке. Рассмотрим узел

$$
\gamma=\frac{1}{2}\left(\gamma^{(1)}-\gamma^{(2)}+\gamma^{(3)}\right)=\left(0, \frac{1}{2}\left(a_{2}+b_{2}-c_{2}\right), \frac{1}{2}\left(a_{3}+b_{3}+c_{3}\right)\right) .
$$

Предположим, что $a_{3}+b_{3}<c_{3}$. Тогда

$$
\gamma_{1}=0, \quad-|S|_{2}<\gamma_{2} \leqslant|S|_{2}, \quad 0 \leqslant \gamma_{3}<|S|_{3}
$$


Поскольку $S$ минимальна, то $\gamma_{2}=|S|_{2}$. А это возможно только для $a_{2}=b_{2}$ и $c_{2}=0$. Из минимальности узлов $\gamma^{(1)}$ и $\gamma^{(2)}$ следует, что $a_{3}=b_{3}$ и $a_{3}<c_{3} / 2$.

Так как $\gamma^{(1)}$ и $\gamma^{(3)}-$ смежные узлы, то существует $\widetilde{\gamma}^{(2)} \in \Gamma$, дополняюший $\gamma^{(1)}$ и $\gamma^{(3)}$ до базиса решетки Г (см. [2] и [4]). Выразим этот узел через $\gamma^{(1)}, \gamma^{(2)}, \gamma^{(3)}$ в следующем виде:

$$
\widetilde{\gamma}^{(2)}=\frac{1}{k}\left(\gamma^{(2)}-n \gamma^{(1)}-m \gamma^{(3)}\right)
$$

с цельми $n, m, k$ и $k>0$. Из условия (1.3) следует, что $k \neq 1$. Обозначим через $u$ и $v$ ближайшие целые к $n / k, m / k$ соответственно и положим $\alpha=n / k-u, \beta=m / k-v$. При этом $-1 / 2 \leqslant \alpha, \beta \leqslant 1 / 2$. Тогда для координат узла

$$
\gamma=\widetilde{\gamma}^{(2)}+u \gamma^{(1)}+v \gamma^{(3)}=\left(a_{1}\left(\frac{1}{k}-\alpha\right),-a_{2}\left(\frac{1}{k}+\alpha\right),-a_{3}\left(\frac{1}{k}+\alpha\right)-c_{3} \beta\right)
$$

при $k \geqslant 3$ выполняются неравенства

$$
\begin{gathered}
\left|\gamma_{1}\right| \leqslant\left(\frac{1}{k}+\frac{1}{2}\right) a_{1}<a_{1}, \quad\left|\gamma_{2}\right| \leqslant\left(\frac{1}{k}+\frac{1}{2}\right) a_{2}<a_{2}, \\
\left|\gamma_{3}\right| \leqslant\left(\frac{1}{k}+\frac{1}{2}\right) a_{3}+\frac{c_{3}}{2} \leqslant\left(\frac{1}{2 k}+\frac{1}{4}+\frac{1}{2}\right) c_{3}<c_{3} .
\end{gathered}
$$

То есть ненулевой узел $\gamma$ лежит внутри $\mathscr{P}\left(|S|_{1},|S|_{2},|S|_{3}\right)$, что противоречит минимальности множества $S$. Таким образом, $k=2$ и в качестве $\widetilde{\gamma}^{(2)}$ можно взять узел $\left(\gamma^{(1)}+\right.$ $\left.\gamma^{(2)}+\gamma^{(3)}\right) / 2$. Поэтому решетка Г порождается базисными узлами

$$
\left(a_{1}, a_{2}, a_{3}\right), \quad\left(a_{1}, 0, \frac{1}{2} c_{3}\right), \quad\left(0,0, c_{3}\right)
$$

и при этом

$$
S=\left\{\left(a_{1}, a_{2}, a_{3}\right),\left(a_{1},-a_{2},-a_{3}\right),\left(0,0, c_{3}\right)\right\} .
$$

Согласно теореме $1 a_{3}<c_{3} / 4$ и $S$ относится к “исключительным" системам.

Если $a_{2}+b_{2}<c_{2}$, то, переставив вторые и третьи координаты, а также умножив $\gamma^{(3)}$ на -1, приходим к случаю, изложенному вьше. Осталось рассмотреть системы $S$ с условиями

$$
a_{2}+b_{2} \geqslant c_{2}, \quad a_{3}+b_{3} \geqslant c_{3} .
$$

Учитьвая минимальность $\gamma^{(1)}$ и $\gamma^{(2)}$, а также принимая во внимание возможность перестановки местами второй и третьей координат вместе с переменой знака у $\gamma^{(3)}$, можно считать, что $a_{2} \geqslant b_{2}$ и $a_{3} \leqslant b_{3}$, причем равенства вьполняются только одновременно.

Пусть $a_{2}=b_{2}$ и $a_{3}=b_{3}$. Рассмотрим узел

$$
\widetilde{\gamma}=\frac{1}{2}\left(\gamma^{(1)}+\gamma^{(2)}+\gamma^{(3)}\right)=\left(a_{1},-\frac{1}{2} c_{2}, \frac{1}{2} c_{3}\right) .
$$

Из минимальности $\gamma^{(1)}$ и (1.4) следует, что $c_{2}=2 a_{2}$ и $c_{3}=2 a_{3}$. В этом случае $\widetilde{\gamma}=$ $\left(a_{1},-a_{2}, a_{3}\right)$ и узел $\gamma^{(1)}-\widetilde{\gamma}+\gamma^{(3)}=\left(0,0, c_{3}\right)$ нарушает минимальность $\gamma^{(3)}$. Таким образом, $a_{2} \neq b_{2}, a_{3} \neq b_{3}$. Следовательно,

$$
a_{2}>b_{2}, \quad a_{3}<b_{3}
$$


Рассмотрим узел

$$
\gamma=\frac{1}{2}\left(\gamma^{(1)}+\gamma^{(2)}+\gamma^{(3)}\right)=\left(a_{1}, \frac{1}{2}\left(a_{2}-b_{2}-c_{2}\right), \frac{1}{2}\left(a_{3}-b_{3}+c_{3}\right)\right) .
$$

Очевидно, что $\gamma_{2}<a_{2}$. В соответствии с (1.4) и (1.5)

$$
\gamma_{2} \geqslant \frac{1}{2}\left(c_{2}-b_{2}-b_{2}-c_{2}\right)=-b_{2}>-a_{2}
$$

Итак, $\left|\gamma_{2}\right|<a_{2}$. Из второго неравенства (1.4) следует, что

$$
\gamma_{3}=\frac{1}{2}\left(a_{3}-b_{3}+c_{3}\right) \leqslant a_{3}
$$

По причине минимальности $\gamma^{(1)}$ вьполняется неравенство

$$
\gamma_{3}=\frac{1}{2}\left(a_{3}-b_{3}+c_{3}\right)<-a_{3}
$$

То есть $3 a_{3}+c_{3}<b_{3}$. Для узла

$$
\gamma=\gamma^{(1)}+\gamma^{(3)}=\left(a_{1}, a_{2}-c_{2}, a_{3}+c_{3}\right)
$$

с $0<\gamma_{3}=a_{3}+c_{3} \leqslant 3 a_{3}+c_{3}<b_{3}$ ввиду минимальности $\gamma^{(2)}$ с учетом (1.4) получаем неравенство $\gamma_{2}=a_{2}-c_{2}>b_{2}$. Тогда

$\gamma_{1}^{(3)}=0<|S|_{1}, \quad\left|\gamma_{2}^{(3)}\right|=c_{2} \leqslant c_{2}+b_{2}<a_{2}=|S|_{2}, \quad\left|\gamma_{3}^{(3)}\right|=c_{3} \leqslant 3 a_{3}+c_{3}<b_{3}=|S|_{3}$.

Таким образом, $\gamma^{(3)}$ нарушает минимальность $S$. Поэтому минимальных систем $S$ с условием (1.4) не существует. Лемма 1 доказана.

\section{2. Доказательство теоремы 2.}

ЛЕмма 2. Пусть $\alpha_{1}, \alpha_{2}, \alpha_{3}$ - вещественные числа $u\left\|\alpha_{1}\right\| \leqslant\left\|\alpha_{2}\right\| \leqslant\left\|\alpha_{3}\right\|$. Тогда

a) $\min \left\{\left\|\alpha_{1}\right\|+\left\|\alpha_{2}\right\|+\left\|\alpha_{3}\right\|,\left\|2 \alpha_{1}\right\|+\left\|2 \alpha_{2}\right\|+\left\|2 \alpha_{3}\right\|\right\} \leqslant 1$;

б) если $\min \left\{\left\|\alpha_{1}\right\|+\left\|\alpha_{2}\right\|+\left\|\alpha_{3}\right\|,\left\|2 \alpha_{1}\right\|+\left\|2 \alpha_{2}\right\|+\left\|2 \alpha_{3}\right\|\right\}=1$, mo

$$
\left\|3 \alpha_{1}\right\|+\left\|3 \alpha_{2}\right\|+\left\|3 \alpha_{3}\right\| \leqslant 1
$$

и равенство достигается только в случае $\left\|\alpha_{1}\right\|=\left\|\alpha_{2}\right\|=1 / 4,\left\|\alpha_{3}\right\|=1 / 2$.

ДокАЗАТЕЛЬСТво. По причине четности и периодичности с периодом 1 функции $\beta=\|\alpha\|$ достаточно рассмотреть случай с $0 \leqslant \alpha_{1} \leqslant \alpha_{2} \leqslant \alpha_{3} \leqslant 1 / 2$. Так как $\alpha_{i} \in[0,1 / 2]$, то $\left\|2 \alpha_{i}\right\| \leqslant 1-2 \alpha_{i}$ и $\left\|\alpha_{i}\right\|=\alpha_{i}$. Положим $\alpha=\alpha_{1}+\alpha_{2}+\alpha_{3}$. Тогда

$$
\min \left\{\left\|\alpha_{1}\right\|+\left\|\alpha_{2}\right\|+\left\|\alpha_{3}\right\|,\left\|2 \alpha_{1}\right\|+\left\|2 \alpha_{2}\right\|+\left\|2 \alpha_{3}\right\|\right\} \leqslant \min \{\alpha, 3-2 \alpha\}=1 .
$$

Утверждение а) доказано.

Пусть

$$
\left\|\alpha_{1}\right\|+\left\|\alpha_{2}\right\|+\left\|\alpha_{3}\right\|=\left\|2 \alpha_{1}\right\|+\left\|2 \alpha_{2}\right\|+\left\|2 \alpha_{3}\right\|=1 .
$$


Предположим, что $\alpha_{1} \geqslant 1 / 4$. Условия $\alpha_{1} \leqslant \alpha_{2} \leqslant \alpha_{3}$ и (2.1) накладьвают ограничения на $\alpha_{1}, \alpha_{2}, \alpha_{3}$ :

$$
\frac{1}{4} \leqslant \alpha_{1} \leqslant \frac{1}{3}, \quad \frac{1}{4} \leqslant \alpha_{2} \leqslant \frac{1}{2}, \quad \frac{1}{3} \leqslant \alpha_{3} \leqslant \frac{1}{2} .
$$

Для этих значений $\alpha_{1}, \alpha_{2}, \alpha_{3}$

$$
\left\|3 \alpha_{1}\right\|+\left\|3 \alpha_{2}\right\|+\left\|3 \alpha_{3}\right\|=\left\{\begin{array}{c}
-2+6 \alpha_{3}, \text { если } \frac{3}{4} \leqslant 3 \alpha_{2} \leqslant 1, \\
2-6 \alpha_{1}, \text { если } 1<3 \alpha_{2} \leqslant \frac{3}{2} .
\end{array}\right.
$$

Отсюда следует, что $\left\|3 \alpha_{1}\right\|+\left\|3 \alpha_{2}\right\|+\left\|3 \alpha_{3}\right\| \leqslant 1$ и равенство достигается только в случае $\alpha_{1}=\alpha_{2}=1 / 4, \alpha_{3}=1 / 2$.

Пусть теперь $0 \leqslant \alpha_{1}<1 / 4$. Тогда, учитывая $(2.1)$ и условие $\alpha_{1} \leqslant \alpha_{2} \leqslant \alpha_{3}$, получаем $2 \alpha_{3} \geqslant \alpha_{2}+\alpha_{3}=1-\alpha_{1}>3 / 4$. Следовательно, $3 / 8<\alpha_{3} \leqslant 1 / 2$. Из (2.1) находим, что $1=\left\|2 \alpha_{1}\right\|+\left\|2 \alpha_{2}\right\|+\left\|2 \alpha_{3}\right\|=2 \alpha_{1}+\left\|2 \alpha_{2}\right\|+1-2 \alpha_{3}$. Поэтому $\left\|2 \alpha_{2}\right\|=2 \alpha_{3}-2 \alpha_{1}$. Далее находим, что

$$
\left\|2 \alpha_{2}\right\|+2 \alpha_{2}=2 \alpha_{3}-2 \alpha_{1}+2 \alpha_{2}=2\left(1-\alpha_{1}\right)-2 \alpha_{1}=2-4 \alpha_{1}>1 .
$$

Это неравенство не выполняется для $0 \leqslant \alpha_{2}<1 / 4$. Поэтому $1 / 4 \leqslant \alpha_{2} \leqslant 1 / 2$. Но тогда

$$
2-4 \alpha_{1}=\left\|2 \alpha_{2}\right\|+2 \alpha_{2}=1-2 \alpha_{2}+2 \alpha_{2}=1 .
$$

То есть $\alpha_{1}=1 / 4$. А это противоречит предположению о том, что $0 \leqslant \alpha_{1}<1 / 4$. Лемма 2 доказана.

ЛЕмма 3. Пусть $S=\left\{\gamma^{(1)}, \gamma^{(2)}, \gamma^{(3)}\right\}-$ минимальная система узлов решетки $\Gamma \in \mathfrak{L}_{3}(\mathbb{R})$. Тогда

$$
\frac{1}{2}\left(\gamma^{(1)} \pm \gamma^{(2)}\right) \notin \Gamma
$$

ДокАЗАТЕЛЬСтво. Заметим, что $\left(\gamma^{(1)}+\gamma^{(2)}\right) / 2$ и $\left(\gamma^{(1)}-\gamma^{(2)}\right) / 2$ только одновременно могут быть узлами $Г$. Предположим, что $\gamma=\left(\gamma^{(1)}+\gamma^{(2)}\right) / 2 \in \Gamma$. Так как $\left|\gamma_{i}\right| \leqslant|S|_{i}$ для всех $i=1,2,3$ и $S$ - минимальная система, то возможен только один вариант: хотя бы по одной координате $\left|\gamma_{i}\right|=|S|_{i} \neq 0$. Без ограничения общности можно считать, что $i=1$ и $\gamma_{1}^{(1)}=\gamma_{1}^{(2)}>0$. Так как $\gamma \in \Gamma$, то

$$
\widetilde{\gamma}=\frac{1}{2}\left(\gamma^{(1)}-\gamma^{(2)}\right)=\left(0, \widetilde{\gamma}_{2}, \widetilde{\gamma}_{3}\right) \in \Gamma
$$

c $\left|\widetilde{\gamma}_{2}\right| \leqslant|S|_{2},\left|\widetilde{\gamma}_{3}\right| \leqslant|S|_{3}$. Для $\widetilde{\gamma}$ также необходимо выполнение хотя бы одного из равенств: $\left|\widetilde{\gamma}_{2}\right|=|S|_{2} \neq 0,\left|\widetilde{\gamma}_{3}\right|=|S|_{3} \neq 0$. Можно считать, что $\left|\widetilde{\gamma}_{2}\right|=|S|_{2}$. В этом случае $\left|\gamma_{2}^{(1)}\right|=\left|\gamma_{2}^{(2)}\right|$. Так как $\gamma^{(1)}, \gamma^{(2)} \in \mathfrak{M}_{\Gamma}$, то и $\left|\gamma_{3}^{(1)}\right|=\left|\gamma_{3}^{(2)}\right|$. Но тогда $\mathscr{P}(\widetilde{\gamma}) \subset \mathscr{P}\left(\gamma^{(1)}\right)=\mathscr{P}\left(\gamma^{(2)}\right)$, что противоречит минимальности $\gamma^{(1)}$ и $\gamma^{(2)}$. Следовательно, наше предположение неверно. Лемма 3 доказана.

Лемма 4. Пусть множсество $S=\left\{\gamma^{(1)}, \gamma^{(2)}, \gamma^{(3)}\right\}-$ минимальная система узлов решетки $\Gamma \in \mathfrak{L}_{3}(\mathbb{R})$, отличная от “исключительной" (см. введение). Тогда для любой комбинации знаков

$$
\frac{1}{2}\left( \pm \gamma^{(1)} \pm \gamma^{(2)} \pm \gamma^{(3)}\right) \notin \Gamma .
$$


ДокАЗАТЕЛЬСтво. Предположим, что утверждение леммы неверно и $\gamma-$ узел решетки Г вида (2.2). Тогда остальные комбинации также являются узлами решетки. Без ограничения общности можно считать, что координаты узла $\gamma^{(1)}$ - неотрицательные числа.

Пусть $i \in\{2,3\}$ и $j \in\{1,2,3\}$. Положим $\varepsilon_{j}^{(i)}=1$, если $\gamma_{j}^{(i)} \geqslant 0$, и $\varepsilon_{j}^{(i)}=-1$, если $\gamma_{j}^{(i)}<0$. Тогда найдутся $\tau_{2}, \tau_{3} \in\{-1,1\}$ с

$$
\left(\tau_{2}, \tau_{3}\right) \notin\left\{\left(\varepsilon_{1}^{(2)}, \varepsilon_{1}^{(3)}\right),\left(\varepsilon_{2}^{(2)}, \varepsilon_{2}^{(3)}\right),\left(\varepsilon_{3}^{(2)}, \varepsilon_{3}^{(3)}\right)\right\} .
$$

Поэтому для

$$
\gamma=\frac{1}{2}\left(\gamma^{(1)}+\tau_{2} \gamma^{(2)}+\tau_{3} \gamma^{(3)}\right)
$$

выполняются неравенства

$$
\left|\gamma_{i}\right| \leqslant \max \left\{\gamma_{i}^{(1)},\left|\gamma_{i}^{(2)}\right|,\left|\gamma_{i}^{(3)}\right|\right\}=|S|_{i}
$$

при всех $i=1,2,3$. Если в (2.3) выполняются строгие неравенства, то из минимальности $S$ следует, что $\gamma=(0,0,0)$. Это невозможно, так как $\gamma^{(1)}, \gamma^{(2)}, \gamma^{(3)}-$ линейно независимые узлы.

Предположим, что в (2.3) хотя бы по одной координате $i$ выполняется равенство. Тогда одно из чисел $\left|\gamma_{i}^{(1)}\right|,\left|\gamma_{i}^{(2)}\right|,\left|\gamma_{i}^{(3)}\right|$ - нуль, а два остальных равны. Переставляя (в случае необходимости) местами координаты с номерами $i$ и 1 , а также принимая во внимание возможность замены знаков по отдельньм координатам и у $\gamma^{(3)}$, мы можем считать, что узлы системы $S$ имеют вид

$$
\gamma^{(1)}=\left(a_{1}, a_{2}, a_{3}\right), \quad \gamma^{(2)}=\left(a_{1}, \varepsilon_{1} b_{2}, \varepsilon_{2} b_{3}\right), \quad \gamma^{(3)}=\left(0, \varepsilon_{3} c_{2}, c_{3}\right)
$$

с положительным $a_{1}$ и неотрицательными $a_{2}, a_{3}, b_{2}, b_{3}, c_{2}, c_{3}$.

Пусть $\varepsilon_{1}=\varepsilon_{2}=\varepsilon_{3}=-1$. Согласно лемме 1 система $S$ является "исключительной". Таким образом, в данном случае исходное предположение неверно.

Пусть $\varepsilon_{1}=-1$ и $\left(\varepsilon_{2}, \varepsilon_{3}\right) \neq(-1,-1)$. Рассмотрим узел

$$
\gamma=\frac{1}{2}\left(\gamma^{(1)}-\gamma^{(2)}-\varepsilon_{3} \gamma^{(3)}\right)=\left(0, \frac{1}{2}\left(a_{2}+b_{2}-c_{2}\right), \frac{1}{2}\left(a_{3}-\varepsilon_{2} b_{3}-\varepsilon_{3} c_{3}\right)\right)
$$

Для его координат справедливы ограничения: $\left|\gamma_{1}\right|<\left|S_{1}\right|,\left|\gamma_{2}\right| \leqslant\left|S_{2}\right|,\left|\gamma_{3}\right| \leqslant\left|S_{3}\right|$. Так как по условию леммы $S$ - минимальная система, то $\gamma^{(1)}, \gamma^{(2)}, \gamma^{(3)}-$ линейно независимые векторы. Поэтому вьполняется хотя бы одно из равенств: $\left|\gamma_{2}\right|=|S|_{2},\left|\gamma_{3}\right|=|S|_{3}$.

Пусть $\left|\gamma_{2}\right|=|S|_{2}$. В этом случае система $S$ имеет вид

$$
S=\left\{\left(a_{1}, a_{2}, a_{3}\right),\left(a_{1},-a_{2}, \varepsilon_{2} a_{3}\right),\left(0,0, c_{3}\right)\right\} .
$$

Для $\varepsilon_{2}=-1$ система совпадает с (1.2). Согласно лемме $1 S$ - “исключительная" система. В случае $\varepsilon_{2}=1$, меняя у узлов системы местами первые и вторые координаты, а также умножая $\gamma^{(2)}$ на -1 , опять приходим к (1.2). Таким образом, $\left|\gamma_{2}\right| \neq|S|_{2}$ и $\left|\gamma_{3}\right|=|S|_{3}$. При этом $S$ можно отнести к одному из трех видов:
a) $S=\left\{\left(a_{1}, a_{2}, a_{3}\right),\left(a_{1},-a_{2},-a_{3}\right),\left(0, c_{2}, 0\right)\right\}$;
б) $S=\left\{\left(a_{1}, a_{2}, a_{3}\right),\left(a_{1},-b_{2}, 0\right),\left(0,-c_{2}, a_{3}\right)\right\}$;
в) $S=\left\{\left(a_{1}, a_{2}, 0\right),\left(a_{1},-b_{2}, b_{3}\right),\left(0, c_{2}, b_{3}\right)\right\}$. 
Переставляя местами (в случае необходимости) вторые и третьи координаты, изменяя порядок следования узлов и меняя знак у некоторых координат, с помощью леммы 1 получаем, что во всех случаях $S$ - “исключительная" система, а это противоречит условию леммы.

Пусть $\varepsilon_{1}=1$ и $\varepsilon_{2}=-1$. Меняя местами вторые и третьи координаты у узлов минимальной системы, приходим к случаю, рассмотренному выше.

Пусть $\varepsilon_{1}=1$ и $\varepsilon_{2}=1$. Для узла

$$
\gamma=\frac{1}{2}\left(\gamma^{(1)}-\gamma^{(2)}+\varepsilon_{3} \gamma^{(3)}\right)=\left(0, \frac{1}{2}\left(a_{2}-b_{2}+c_{2}\right), \frac{1}{2}\left(a_{3}-b_{3}+\varepsilon_{3} c_{3}\right)\right)
$$

имеют место ограничения: $\left|\gamma_{1}\right|<|S|_{1}, \gamma_{2} \leqslant|S|_{2},\left|\gamma_{3}\right| \leqslant|S|_{3}$. Как отмечалось вьше, для того, чтобы не нарушались условия леммы, необходимо вьполнение хотя бы одного из равенств: $\left|\gamma_{2}\right|=|S|_{2},\left|\gamma_{3}\right|=|S|_{3}$. А это возможно только для систем вида

a) $S=\left\{\left(a_{1}, a_{2}, a_{3}\right),\left(a_{1}, 0, b_{3}\right),\left(0, \varepsilon_{3} a_{2}, c_{3}\right)\right\}$;

б) $S=\left\{\left(a_{1}, a_{2}, a_{3}\right),\left(a_{1}, b_{2}, 0\right),\left(0, c_{2}, a_{3}\right)\right\}$;

в) $S=\left\{\left(a_{1}, a_{2}, 0\right),\left(a_{1}, b_{2}, b_{3}\right),\left(0,-c_{2}, b_{3}\right)\right\}$.

Система а) - частный случай $S$ с $\varepsilon_{1}=-1$. Такую ситуацию мы обсуждали вьше. Система б) перестановкой вторых и третьих координат сводится к виду а). Система в) преобразуется к виду б), если поменять местами $\gamma^{(1)}, \gamma^{(2)}$ и изменить знак у $\gamma^{(3)}$. Следовательно, $S$ - “исключительная, система, чего не может быть. Лемма 4 доказана.

Лемма 5. Пусть $S=\left\{\gamma^{(1)}, \gamma^{(2)}, \gamma^{(3)}\right\}$ - минимальная система узлов решетки $\Gamma \in \mathfrak{L}_{3}(\mathbb{R})$. Тогда для любой комбинации знаков

$$
\frac{1}{3}\left( \pm \gamma^{(1)} \pm \gamma^{(2)}\right) \notin \Gamma \text {. }
$$

ДокАЗАТЕЛЬСтво. Предположим, что $\gamma=\left( \pm \gamma^{(1)} \pm \gamma^{(2)}\right) / 3 \in \Gamma$. Для координат узла справедливо ограничение $\left|\gamma_{i}\right| \leqslant 2|S|{ }_{i} / 3$, что противоречит минимальности $S$. Таким образом, $\left( \pm \gamma^{(1)} \pm \gamma^{(2)}\right) / 3 \notin \Gamma$. Лемма 5 доказана.

ЛЕмма 6. Пусть $S=\left\{\gamma^{(1)}, \gamma^{(2)}, \gamma^{(3)}\right\}-$ минимальная система узлов решетки $\Gamma \in \mathfrak{L}_{3}(\mathbb{R})$. Тогда для любой из восьми комбинаций знаков

$$
\frac{1}{3}\left( \pm \gamma^{(1)} \pm \gamma^{(2)} \pm \gamma^{(3)}\right) \notin \Gamma
$$

ДокАЗАтЕЛЬСтво. Пусть $\gamma=\left( \pm \gamma^{(1)} \pm \gamma^{(2)} \pm \gamma^{(3)}\right) / 3 \in \Gamma$. Для $i=1,2,3$ выполняется неравенство $\left|\gamma_{i}\right| \leqslant|S|_{i}$. Так как $S$ - минимальная система, то для одной из координат $\left|\gamma_{i}\right|=|S|_{i} \neq 0$. Без ограничения обшности можно считать, что $\gamma_{1}^{(1)}=\gamma_{1}^{(2)}=\gamma_{1}^{(3)}=t>0$ и

$$
\gamma=\frac{1}{3}\left(\gamma^{(1)}+\gamma^{(2)}+\gamma^{(3)}\right) \in \Gamma
$$

Так как для любого $j=1,2,3$ не выполняется включение $\mathscr{P}(\gamma) \subset \mathscr{P}\left(\gamma^{(j)}\right)$, то $\gamma_{2} \neq 0$ и $\gamma_{3} \neq 0$. При этом можно считать, что $\gamma_{2}>0$ и $\gamma_{3}>0$. Если все координаты $\gamma^{(j)}$ неотрицательны, то узел $\gamma^{(j)}-\gamma$ нарушает условие минимальности $S$. Поэтому у любого узла из $S$ найдется отрищательная координата (вторая или третья). Поскольку $\gamma_{2}$ и $\gamma_{3}-$ 
положительные числа, то найдутся узлы с положительной второй и с положительной третьей координатой.

Предположим, что $\gamma_{2}^{(1)} \gamma_{2}^{(2)}$ и $\gamma_{3}^{(1)} \gamma_{3}^{(2)}$ - неотрицательные числа. Рассмотрим ненулевой узел

$$
\widetilde{\gamma}=\gamma^{(1)}-\gamma^{(2)}=\left(0, \gamma_{2}^{(1)}-\gamma_{2}^{(2)}, \gamma_{3}^{(1)}-\gamma_{3}^{(2)}\right)
$$

Если $\gamma_{2}^{(1)}, \gamma_{2}^{(2)}, \gamma_{3}^{(1)}, \gamma_{3}^{(2)}$ - числа, не равные нулю, то $\widetilde{\gamma}$ лежит внутри $\mathscr{P}\left(|S|_{1},|S|_{2},|S|_{3}\right)$, что противоречит минимальности множества $S$. Следовательно, выполняется хотя бы одно равенство: $\gamma_{2}^{(1)} \gamma_{2}^{(2)}=0, \gamma_{3}^{(1)} \gamma_{3}^{(2)}=0$. Без ограничения общности можно считать, что $\gamma_{3}^{(1)}=0$. Так как у каждого узла есть отрицательная координата и $\gamma_{2}^{(1)} \gamma_{2}^{(2)} \geqslant 0$, то $\gamma_{2}^{(1)}<0, \gamma_{2}^{(2)} \leqslant 0$. Кроме того, в $S$ сушествует узел с положительной второй координатой. Поэтому $\gamma_{2}^{(3)}>0$. Тогда для координат узла

$$
\widetilde{\gamma}=\gamma-\gamma^{(2)}=\left(0, \frac{1}{3}\left(\gamma_{2}^{(1)}-2 \gamma_{2}^{(2)}+\gamma_{2}^{(3)}\right), \frac{1}{3}\left(-2 \gamma_{3}^{(2)}+\gamma_{3}^{(1)}\right)\right)
$$

справедливы следующие оценки:

$$
\begin{gathered}
\left|\widetilde{\gamma}_{1}\right|<|S|_{1}, \\
-|S|_{2}<\widetilde{\gamma}_{2}=\frac{1}{3}\left(-\left|\gamma_{2}^{(1)}\right|+2\left|\gamma_{2}^{(2)}\right|+\gamma_{2}^{(3)}\right)<\frac{1}{3}\left(2\left|\gamma_{2}^{(2)}\right|+\left|\gamma_{2}^{(3)}\right|\right) \leqslant|S|_{2}, \\
\left|\widetilde{\gamma}_{3}\right|=\frac{1}{3}\left(2\left|\gamma_{3}^{(2)}\right|+\left|\gamma_{3}^{(3)}\right|\right) \leqslant|S|_{3} .
\end{gathered}
$$

Из минимальности $S$ следует, что $\left|\gamma_{3}^{(2)}\right|=\left|\gamma_{3}^{(3)}\right|$ и $\gamma_{3}^{(2)} \gamma_{3}^{(3)}<0$. В этом случае $\gamma_{3}=0$. Ранее мы установили, что $\gamma_{3}>0$. Таким образом, ни у одного из узлов множества $S$ знаки координат не совпадают. Без ограничения общности можно считать, что

$$
\gamma^{(1)}=\left(t, a_{2},-a_{3}\right), \quad \gamma^{(2)}=\left(t,-b_{2}, b_{3}\right), \gamma^{(3)}=\left(t,-c_{2},-c_{3}\right)
$$

с неотрищательньми числами $a_{2}, a_{3}, b_{2}, b_{3}, c_{2}, c_{3}$.

Рассмотрим узел

$$
\widetilde{\gamma}=\gamma-\gamma^{(3)}=\frac{1}{3}\left(-2 \gamma^{(3)}+\gamma^{(1)}+\gamma^{(2)}\right)=\left(0, \frac{1}{3}\left(a_{2}-b_{2}+2 c_{2}\right), \frac{1}{3}\left(-a_{3}+b_{3}+2 c_{3}\right)\right) .
$$

Узел $\widetilde{\gamma}$ не нарушает минимальность $S$ только для $b_{2}=0, c_{2}=a_{2}, c_{3}=a_{3}$. Тогда система имеет вид

$$
S=\left\{\left(t, a_{2},-a_{3}\right),\left(t, 0, b_{3}\right),\left(t,-a_{2},-a_{3}\right)\right\} .
$$

И в этом случае получили противоречие с тем, что $\gamma_{2}>0$. Следовательно, наше предположение о том, что узел вида $\left(\gamma^{(1)}+\gamma^{(2)}+\gamma^{(3)}\right) / 3 \in \Gamma$ неверно. Лемма 6 доказана.

Перейдем к доказательству теоремы 2.

Обозначим через $\widetilde{\Gamma}$ подрешетку в $\Gamma$, порожденную узлами $\gamma^{(1)}, \gamma^{(2)}, \gamma^{(3)}$. Так как $\gamma^{(1)}$ и $\gamma^{(2)}$ - смежные минимумы, существует узел $\gamma \in \Gamma$, дополняющий $\gamma^{(1)}$ и $\gamma^{(2)}$ до базиса решетки (см. [2] и [4]). Выразим $\gamma, 2 \gamma, 3 \gamma$ через $\gamma^{(1)}, \gamma^{(2)}, \gamma^{(3)}$ :

$$
\begin{gathered}
\gamma=\alpha_{1} \gamma^{(1)}+\alpha_{2} \gamma^{(2)}+\alpha_{3} \gamma^{(3)}, \quad 2 \gamma=2 \alpha_{1} \gamma^{(1)}+2 \alpha_{2} \gamma^{(2)}+2 \alpha_{3} \gamma^{(3)} \\
3 \gamma=3 \alpha_{1} \gamma^{(1)}+3 \alpha_{2} \gamma^{(2)}+3 \alpha_{3} \gamma^{(3)}
\end{gathered}
$$


с $\alpha_{1}, \alpha_{2}, \alpha_{3} \in \mathbb{R}$. Обозначим через $a_{i}(k)$ ближайшие целые к $k \alpha_{i}$ и рассмотрим узлы решетки следующего вида:

$$
\widetilde{\gamma}^{(k)}=k \gamma-a_{1}(k) \gamma^{(1)}-a_{2}(k) \gamma^{(2)}-a_{3}(k) \gamma^{(3)}=r_{1}(k) \gamma^{(1)}+r_{2}(k) \gamma^{(2)}+r_{3}(k) \gamma^{(3)},
$$

где $k \alpha_{i}=a_{i}(k)+r_{i}(k) \mathrm{c}-1 / 2 \leqslant r_{i}(k)<1 / 2$. Тогда

$$
\left|\widetilde{\gamma}_{i}^{(1)}\right| \leqslant|S|_{i}\left(\left\|\alpha_{1}\right\|+\left\|\alpha_{2}\right\|+\left\|\alpha_{3}\right\|\right), \quad\left|\widetilde{\gamma}_{i}^{(2)}\right| \leqslant|S|_{i}\left(\left\|2 \alpha_{1}\right\|+\left\|2 \alpha_{2}\right\|+\left\|2 \alpha_{3}\right\|\right) .
$$

Согласно лемме 2

$$
\min \left\{\left\|\alpha_{1}\right\|+\left\|\alpha_{2}\right\|+\left\|\alpha_{3}\right\|,\left\|2 \alpha_{1}\right\|+\left\|2 \alpha_{2}\right\|+\left\|2 \alpha_{3}\right\|\right\} \leqslant 1
$$

Если здесь вьполняется строгое неравенство, то хотя бы один из узлов $\widetilde{\gamma}^{(1)}, \widetilde{\gamma}^{(2)}$ лежит внутри $\mathscr{P}(S)$. Такой узел по причине минимальности $S$ совпадает с $(0,0,0)$.

Если $\widetilde{\gamma}^{(1)}=(0,0,0)$, то $\gamma=a_{1}(1) \gamma^{(1)}+a_{2}(1) \gamma^{(2)}+a_{3}(1) \gamma^{(3)}$ и $\gamma^{(1)}, \gamma^{(2)}, \gamma^{(3)}$ составляют базис $\Gamma$. Поэтому $\widetilde{\Gamma}=\Gamma$.

Предположим теперь, что $\widetilde{\gamma}^{(1)} \neq(0,0,0)$. Тогда $\widetilde{\gamma}^{(2)}=(0,0,0)$ и $\gamma=a_{1}(2) \gamma^{(1)} / 2+$ $a_{2}(2) \gamma^{(2)} / 2+a_{3}(2) \gamma^{(3)} / 2$. Положив $b_{j}(2)=2 a_{j}(2)+\varepsilon_{j}$ с целыми $b_{j}(2)$ и $\varepsilon_{j} \in\{0,1\}$, получаем, что узел $\left(\varepsilon_{1} \gamma^{(1)}+\varepsilon_{2} \gamma^{(2)}+\varepsilon_{3} \gamma^{(3)}\right) / 2$ является узлом решетки. А это противоречит леммам 3 и 4 .

Предположим, что

$$
\min \left\{\left\|\alpha_{1}\right\|+\left\|\alpha_{2}\right\|+\left\|\alpha_{3}\right\|,\left\|2 \alpha_{1}\right\|+\left\|2 \alpha_{2}\right\|+\left\|2 \alpha_{3}\right\|\right\}=1 .
$$

Согласно лемме $2\left\|3 \alpha_{1}\right\|+\left\|3 \alpha_{2}\right\|+\left\|3 \alpha_{3}\right\| \leqslant 1$, причем равенство достигается только в случае, когда два из чисел $\left\|\alpha_{1}\right\|,\left\|\alpha_{2}\right\|,\left\|\alpha_{3}\right\|$ равны $1 / 4$, а третье $-1 / 2$. При строгом неравенстве ввиду минимальности $S \widetilde{\gamma}^{(3)}=(0,0,0)$. Отсюда следует, что $\left(\varepsilon_{1} \gamma^{(1)}+\varepsilon_{2} \gamma^{(2)}+\right.$ $\left.\varepsilon_{3} \gamma^{(3)}\right) / 3 \mathrm{c} \varepsilon_{i} \in\{-1,0,1\}$ является узлом решетки $Г$. Но это противоречит утверждениям лемм 5 и 6 .

Осталось рассмотреть случай, когда с точностью до перестановки узлов $S$ вьполняются равенства $\left\|\alpha_{1}\right\|=1 / 4,\left\|\alpha_{2}\right\|=1 / 4,\left\|\alpha_{3}\right\|=1 / 2$. Тогда $\widetilde{\gamma}=\gamma^{(1)} / 4+\gamma^{(2)} / 4+\gamma^{(3)} / 2$ является узлом решетки. Следовательно, $2 \widetilde{\gamma}=\gamma^{(1)} / 2+\gamma^{(2)} / 2+\gamma^{(3)} \in \Gamma$, что противоречит утверждению леммы 3 . Таким образом, $\widetilde{\Gamma}=\Gamma$. Теорема 2 доказана.

Автор признательна В. А. Быковскому за постановку задачи и внимание.

\section{СПИСОК ЦИТИРОВАННОЙ ЛИТЕРАТУРЫ}

[1] Касселс Дж. Введение в геометрию чисел. М.: Мир, 1965.

[2] Вороной Г.Ф. // Собрание сочинений. Т. 1. Киев: Наукова думка, 1952. С. 197-391.

[3] Minkowski H. Zur Theorie der Kettenbrüche // Gesammelte Abhandlungen. Leipzig, 1911. P. 278-292.

[4] Быковский В. А. Теорема Валена для двумерных подходящих дробей // Матем. заметки. 1999. Т. 66. №1. С. 30-37.

Хабаровское отделение Института прикладной математики ДВО РАН

E-mail: gorkusha@iam.khv.ru 